Accretion Phenomena and Related Outflows, IAU Colloquium 163

ASP Conference Series, Vol. 121, 1997

D.T. Wickramasinghe, L. Ferrario, and G.V. Bicknell, eds.

\title{
A Model of Hybrid Magnetospheric Structure Filled with Cylindrical Polar Jet and Conical Wind Envelope
}

\author{
Shin-ya Nitta \\ Department of Applied Physics, Nagoya University, Nagoya 464-01, \\ JAPAN
}

\section{Introduction}

Non force-free cylindrical equilibrium of the magnetically driven relativistic MHD flows is investigated as a model of astrophysical jets/winds. This work is a generalization of the previous work Nitta (1995). We consider the situation that the magnetic field lines emanated from the central object are rotating, and the frozen plasmas are magnetically accelerated outward and construct a selforganized magnetosphere due to the Lorentz force and the inertial force. Recent observations revealed that many objects have a hybrid structure of collimated jet and non-collimated wind regardless with species of the central object, e.g. YSO's, stars, pulsars or AGN's. In order to explain these structures, we present a model that the magnetosphere has a hybrid asymptotic structure: the cylindrical jet and the conical wind fill the infinitely distant polar region and the lower latitude region, respectively. The asymptotically conical wind was investigated in the previous paper (Nitta 1994). Here we investigate the asymptotically cylindrical jet at 'finite cylindrical radius' from the rotational axis. In this case, the inertial force is important for the force balance because a fraction of the flows having finite angular momentum converges to finite cylindrical radius.

\section{Polar Equation}

We suppose relativistic, pressure-free, inviscid and resistive-less MHD flows, and ideal symmetry, i.e. stationary and axisymmetric flows around the rotational axis of the central object. In this case, MHD equations reduce to well known two basic equations for 2-D problem called the Bernoulli equation (BE) and the Grad-Shafranov equation (GSE) with four flux constants: the energy $E$, the angular momentum $L$, the particle injection rate $\eta$ and the angular velocity $\Omega_{F}$ of the field line. We replace the energy $E$ to more convenient one: the terminal velocity $u_{f}$ of the flow. In this paper, our discussion is restricted to the polar region far from the equatorial plane. In order to discuss the connection with the conical wind at the asymptotic region, our discussion must include the region $R \rightarrow \infty$, where $R$ is the cylindrical radius. Here we introduce a more convenient variable $x$ instead of $R$ as $R=R_{L} \tan (x \pi / 2)$ where $R_{L}$ is the representative value of the light cylinder radius. In such polar region, BE and GSE can reduce to a second order ordinary differential equation of the magnetic flux function $\Psi(x)$ when we neglect the variability along the rotational axis. We call it the 'polar equation' (see Nitta 1996). We impose the boundary condition $\Psi=0$ at $x=0(R=0)$ and $\Psi=\Psi_{s}$ at $x=1(R=\infty)$. The first condition is trivial 
for non-singular magnetic distribution, and the second condition denotes that the entire polar region is filled with a finite amount of magnetic flux $\Psi_{s}$ which can be produced by the central object. This is important for self-organized magnetosphere. Thus we numerically solve the polar equation.

\section{Numerical Solutions}

The parameters in this problem are $u_{f}(\Psi), L(\Psi), \eta(\Psi)$ and $\Omega_{F}(\Psi)$. In order to match with the conical wind envelope, we should demand that $u_{f}\left(\Psi_{s}\right)=0$ (see Nitta 1996). We adopt a simple model that $u_{f}(\Psi)=u_{0}\left(1-\Psi / \Psi_{s}\right), L(\Psi)=$ $L_{0} \Psi / \Psi_{s}, \eta(\Psi)=\eta_{0}$ and $\Omega_{F}(\Psi)=\Omega_{0}$ as an example, where $u_{0}, L_{0}, \Omega_{0}$ and $\eta_{0}$ are constants. From the figure of the logarithm of the number density $n$ versus the logarithm of $R$, we can clearly find the core-envelope structure of the density profile, i.e. the dense core forms near the rotational axis and connects to the envelope decaying with a power-law. The same result is found, e.g. in the distribution of the current density and the magnetic field.

\section{Summary}

This equilibrium is characterized by the following noteworthy properties. (1)The jet itself has hybrid core-envelope structure. These two components can be distinguished by two different power-laws of distribution. The jet core is confined inside a scale similar to the light cylinder radius. The core radius depends on parameters $u_{f}, L, \eta$ and $\Omega_{F}$. We can find that $u_{f}, L$ and $\eta$ suppress the collimation, however $\Omega_{F}$ enhances it. The jet envelope fills outside the core region and smoothly connects to the conical wind envelope. (2)This cylindrical jet is a self-organized structure. The jet fills the entire polar region with a finite amount of the poloidal magnetic flux which can be produced by the central object. The jet needs no external pressure of ambient medium for the core formation. (3)The electric current distribution is completely regular. This poloidal current is an inevitable result of confinement of the rotating plasma column. The current distribution is important to check whether the solution is physically plausible or not. In this solution, the current density is finite everywhere, and the total current is also finite.

This current carrying cylindrical jet is important also to understand the origin of the polar current. The polar current is necessary to form the conical wind envelope filling the lower latitude region (see Nitta 1994), and to complete the current circuit of the magnetosphere. Here we can clarify the mechanical process of the polar current.

The author would like to present this hybrid model of outflows as a universal model for understanding outflows from various species of the central objects.

\section{References}

Nitta, S. 1994, PASJ, 46, 217

Nitta, S. 1995, MNRAS, 276, 825

Nitta, S. 1996, MNRAS, to be published 